\title{
PRELIMINARY STUDY ON EARTHQUAKE SURFACE RUPTURE EXTRACTION FROM UAV IMAGES
}

\author{
Yuan Xiaoxiang, Wang Xiaoqing* Ding Xiang, Wu Xiaoyong, Dou Aixia, Wang Shumin
}

Institute of Earthquake Forecasting, China Earthquake Administration, Beijing, China;42585936@qq.com

KEY WORDS: UAV, earthquake surface rupture, 3D reconstruction

\begin{abstract}
:
Because of the advantages of low-cost, lightweight and photography under the cloud, UAVs have been widely used in the field of seismic geomorphology research in recent years. Earthquake surface rupture is a typical seismic tectonic geomorphology that reflects the dynamic and kinematic characteristics of crustal movement. The quick identification of earthquake surface rupture is of great significance for understanding the mechanism of earthquake occurrence, disasters distribution and scale. Using integrated differential UAV platform, series images were acquired with accuracy POS around the former urban area (Qushan town) of Beichuan County as the area stricken seriously by the 2008 Wenchuan Ms8.0 earthquake. Based on the multi-view 3D reconstruction technique, the high resolution DSM and DOM are obtained from differential UAV images. Through the shade-relief map and aspect map derived from DSM, the earthquake surface rupture is extracted and analyzed. The results show that the surface rupture can still be identified by using the UAV images although the time of earthquake elapse is longer, whose middle segment is characterized by vertical movement caused by compression deformation from fault planes.
\end{abstract}

\section{INTRODUCTION}

After the devastating earthquake, the intense tectonic movement often results in the surface rupture on a certain scale. The location and spatial distribution of earthquake surface rupture plays an important role in estimating the seismogenic mechanism, tectonic activity and disaster distribution of the earthquake. In the past, earthquake surface rupture was investigated by artificial ground surveys, satellite and aerial image interpretation methods (Xu X. et al, 2008; Fu B. et al, 2008; Ran Y. et al, 2010).

In recent years, with the further development of UAV platform and image processing method base on computer vision, it has become the focus for many scholars to use the high overlap degree of UAV images to reconstruct 3D scenes and use highprecision digital elevation model to study the active tectonics. Bemis S. P. et al. (2014) conducted a study on the tectonics and paleoearthquakes using the three-dimensional model established by UAV images and achieved good results. Taking the Dushanzi reverse fault as an example, Wei Z. et al. (2015) obtained a set of photos from a simple balloon platform and obtained the high-precision topographic information by using the 3D reconstruction technology. Compared with the LiDAR data, Topographic data accuracy is comparable to corresponding LiDAR data. Gao S. et al. (2017) obtained the high-precision topographic information of a fault in the Barkol Basin in Xinjiang by using UAV images and considered it as an effective method to identify the residual tectonic information of complex alluvial fans. The above research results show that the UAV image can obtain high-resolution 3D digital elevation information for active structural information identification and analysis. However, previous structural research based on 3D reconstruction recovery technique need ground control point by accurate measurement, which depress the efficiency greatly for practical earthquake surface rupture interpretation.
Hence, taking the surface rupture near extreme earthquake disaster area (former Beichuan County) caused by Wenchuan Ms 8.0 earthquake as an example, this paper uses the recent acquisition of UAV images with differential POS data and uses the multi-vision $3 \mathrm{D}$ reconstruction technology to obtain the high accuracy of surface terrain information of rupture for extraction research.

\section{MULTI-VIEW 3D RECONSTRUCTION METHOD}

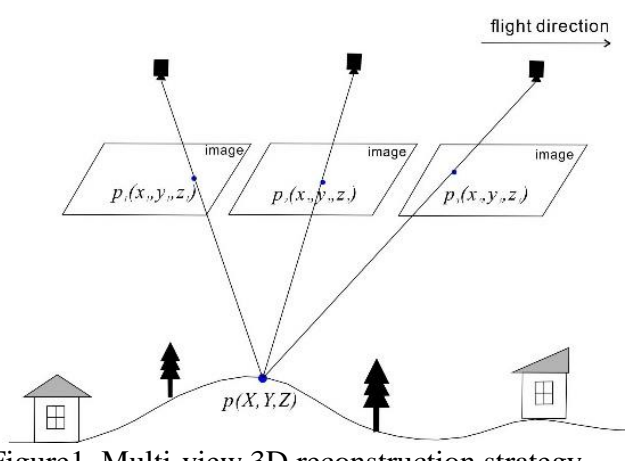

Figure1. Multi-view 3D reconstruction strategy

The multi-view 3D reconstruction method mainly utilizes the highly overlapped photos acquired from different locations to calculate and recover the $3 \mathrm{D}$ information of the object. The specific process includes the structure from motion ( $\mathrm{SfM})$ and multi-view Stereo (MVS) (Snavely N. et al, 2008; James M.R. et al, 2012). The SfM method first identifies the feature points for each image. Then matches the pixels of the same name point with overlapping images. After that the camera parameters of each image are restore to obtain the sparse three-dimensional position of the target (Snavely N. et al, 2010). The specific process shown in Figure 1. The multi-view 3D stereo

\footnotetext{
* Corresponding author

E-mail address: wangxiaoq517@163.com
} 
reconstruction process is based on the initial position obtained by the SfM, and the clustering optimization is performed on the images to obtain a dense point cloud with optimized target (James et al, 2008; Zhang et al, 2015). In this paper, based on SfM and MVS method, we get the dense point clouds of the study area, and then build the terrain 3D model to generate the digital surface model (DSM) (Figure 4a).

\section{UAV DATA ACQUISITION AND EARTHQUAKE SURFACE RUPTURE IDENTIFICATION}

The study area is about $7 \mathrm{~km}$ long and $0.6 \mathrm{~km}$ wide and is located in former urban area of Beichuan County and its surrounding area (Figure 2). After the 5.12 Wenchuan earthquake, a large number of reverse surface ruptures with dextral strike slip component were formed with some reverse fault scarps, compressional nappe scarps and others along the Beichuan-yingxiu fault in this area. There was an obvious vertical movement characteristic of the earthquake surface rupture zone near the Shaba village in Beichuan county (Xu X. et al, 2008; 2009; Tan X. et al, 2010; Ran Y. et al, 2010).

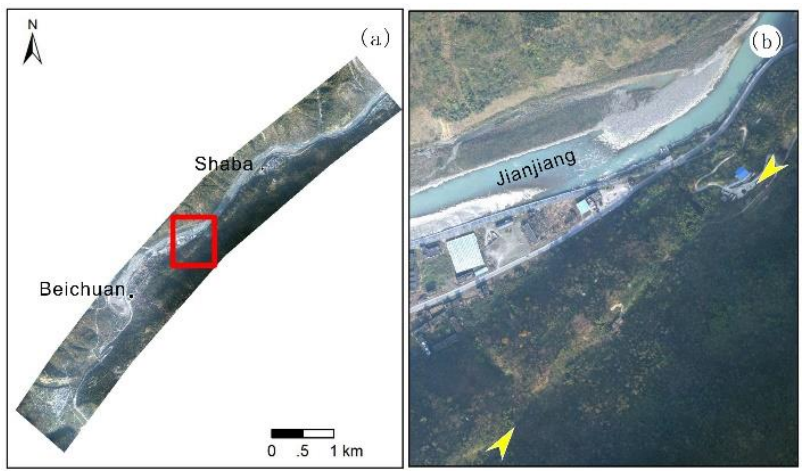

Figure 2. Mosaic image in study area. (a) Ortho image; (b) Zoomed image of red rectangle in (a).

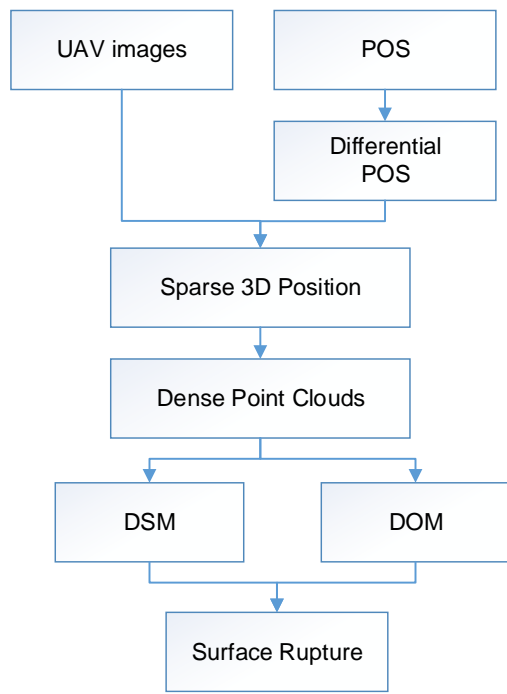

Figure 3. UAV images processing Flow

Using vertical take-off and landing UAV produced by JOUAV Company, a series of UAV images were obtained, whose degree of heading overlap is $80 \%$ and the lateral overlap is $70 \%$. The relative flight height is about $700 \mathrm{~m}$. The single image size is
$7360 \times 4912$ pixels. In order to obtain the relatively highprecision surface geo-information in study area, the differential GPS is used to obtain the POS data of the flight. The mean of the horizontal distance deviation of POS between the difference and non-difference is about $192.7 \mathrm{~cm}$, and the mean of the vertical distance deviation is about $44.1 \mathrm{~m}$. Table 1 show some specific deviation values between pre and post differential POS data. Finally, the average resolution of the generated DOM (Figure 2) is about $0.1 \mathrm{~m}$ and DSM (Figure $4 \mathrm{a}$ ) about $0.2 \mathrm{~m}$. The specific data processing flow chart is showed as Figure 3.

\begin{tabular}{cccc}
\hline \hline Image ID & $\begin{array}{c}\triangle \mathrm{x} \\
(\mathrm{cm})\end{array}$ & $\begin{array}{c}\triangle \mathrm{y} \\
(\mathrm{cm})\end{array}$ & $\begin{array}{c}\triangle \mathrm{z} \\
(\mathrm{m})\end{array}$ \\
\hline DSC_0003 & 136.82 & 148.95 & -44.02 \\
\hline DSC_0004 & 155.85 & 149.97 & -44.19 \\
\hline DSC_0005 & 103.54 & 134.82 & -44.33 \\
\hline DSC_0006 & 135.58 & 185.21 & -44.18 \\
\hline DSC_0007 & 93.55 & 182.42 & -44.26 \\
\hline DSC_0008 & 129.45 & 151.44 & -44.22 \\
\hline DSC_0009 & 117.34 & 172.11 & -44.17 \\
\hline DSC_0010 & 120.21 & 158.80 & -44.24 \\
\hline \hline
\end{tabular}

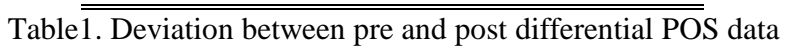

The shaded-releif method of terrain make the terrain contrast characteristic of light and shade distribution through photometric approximate continuous changing hue from simulation of the solar light generated on the ground radiation brightness, and using gray tone or color tone. That makes the distribution, relief and morphological feature of landform have a sense of stereoscopic sense, which intuitively express the terrain.
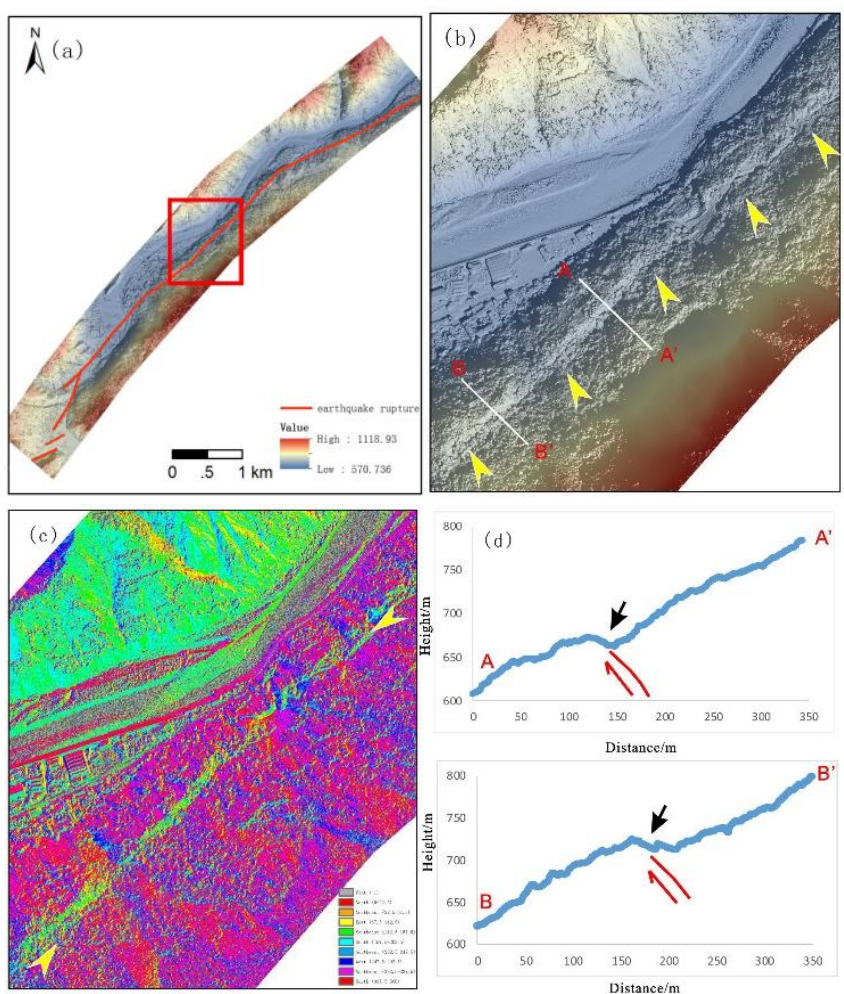

Figure 4. Beichuan topographic map and seismic surface rupture;(a) The shade-relief of the study area; (b) Zoomed 
image of red rectangle in (a); (c) Topographic slope image; (d) the topographic profile cross the seismic surface rupture in (b).

Based on shaded-relief map of study area from generated DSM, intuitionistic and stereoscopic expression of some landform indicators such as fault scarp, surface dislocation and others helps to identify the location of earthquake surface rupture. Through the experiments of different light direction angles and height angles, when the azimuth is 135 degrees and the height angle is 45 degrees, a clear strip shape tonal abnormality is obviously showed between the Shaba village and the former Beichuan County, and the trend is North East (Figure 4b). Based on visual interpretation of linear and terrain features, exact position of the earthquake surface rupture is obtained (Figure 4a, b). Through calculating the aspect image, it shows that the middle segment of main fault faces southeast and east (Figure 4c). It has obvious vertical movement. However, aspects of north segment and south segment are not obvious orientation because of smaller vertical movement. The result is in accordance with previous field survey results (Xu X. et al, 2008; 2009; Tan X. et al, 2010; Ran Y. et al, 2010).

By measuring the cross-section of the fault, the obvious topographic mutation can be seen as shown by the arrows in Figure $4 \mathrm{~d}$. Due to the compression movement between the fault planes, the north-western side increased, resulting in the formation of the fault scarp, which changed the original topography characteristics of low in the northwest and high in the southeast, and formed the obvious terrain anomaly mark.

\section{CONCLUSION AND DISCUSSION}

The results of this study show that although the study area has a thick vegetation cover, higher accuracy DSM can be produced using the UAV image with differential POS data compared to non-differential POS data. According to the terrain analysis of DSM such as shade-relief and aspect, it can be found that the earthquake surface rupture caused some features of topographic change like fault scarp, trough valley et al., which cause obviously linear tone distribution due to movement in vertical and horizontal direction. However, due to the effect of thick vegetation cover on the earthquake-ruptured surface, the horizontal motion characteristics of the earthquake rupture need further study.

\section{ACKNOWLEDGEMENTS}

We thank JOUAV Company for providing UAV images of study area and Beichuan Earthquake Disater Prevention Bureau for providing guidance for study area determination. This work funded by National Key Research and Development Program of China (2017YFB0504104)

\section{REFERENCES}

Bemis S.P., Micklethwaite S., Turner D., et al., 2014. Groundbased and UAV-Based Photogrammetry: A multi-scale, Highresolution Mapping Tool for Structural Geology and Paleoseismology. Journal of Structural Geology, 69, ppt. 163178

Fu B., Shi P., and Zhang Z., 2008. Spatial Characteristics of the Surface Rupture Produced by the Ms 8.0 Wenchuan Earthquake
Using High-resolution Remote Sensing Imagery. Acta Geology Sinica. 82(12), pp. 1679-1687. (In Chinese)

Gao S., Ran Y., Wu F., 2017. et al. Using UAV Photogrammetry Technology to Extract Information of Tectonic Activity of Complex Alluvial Fan- a Case of Study of an Alluvial Fan in the Southern Margin of Barkol Basin. Seismology and geology. 39(4), pp. 793-804, 2017. (In Chinese)

James M.R, and Robson S., 2012. Straightforward Reconstruction of 3D Surfaces and Topography with a Camera: Accuracy and Geoscience Application. Journal of Geophysical Research: Earth Surface, 117(F3), pp. 1-17.

Ran Y., Shi X., Wang H., et al., 2010. The Maximum Coseismic Vertical Surface Displacement and Surface Deformation Pattern Accompanying the Ms 8.0 Wenchuan Earthquake. Chinese Sci Bull, 55(2), pp. 154-162.

Snavely N., Simon I., 2010. Geosele M., et al., Scene Reconstruction and Visualization from Community Photo Collections. Proceedings of the IEEE, 98(8), pp, 1370-1390.

Snavely. N., Seitz S.M. and Szeliski R, 2008. Modeling the World from Internet Photo Collections. International Journal of Computer Vision, 80(2), pp. 189-210.

Tan X. Yuan R. Xu X. et al., 2010. The special characteristics of rupture induced by Ms8.0 Wenchuan earthquake in Leigu area, and its mechanism. Earth Science Frontiers, 17(5), pp, 7583. (In Chinese)

Wei Z., R. Arrow smith, H. He, et al., 2015. Accuracy Analysis of Terrain Point Cloud Acquired by "Structure from Motion" using Aerial Photos. Seismology and geology, 37 (2), pp. 636648. (In Chinese)

Xu X., Wen X., Ye J., et al., 2008. The Ms 8.0 Wenchuan Earthquake Surface Rupture and its Seismogenic Structure. Seismology and geology, 30(3), pp. 597-627. (In Chinese)

Xu X., Wen X., Yu G. et al., 2009. Coseismic reverse- and oblique-slip surface faulting generated by the $2008 \mathrm{Mw} 7.9$ Wenchuan earthquake. China. Geology, 37(6), pp. 515-518.

Zhang P., Wang S., Huang J., et al., 2015. Research on Building Point Cloud Reconstruction Method based on SFM and CMVS/PMVS. Journal of Suzhou University of Science and Technology (Natural Science), 32(3), pp. 60-64. (In Chinese) 